\title{
Legal and policy responses to the delivery of abortion care during COVID-19
}

DOI:

10.1002/ijgo.13377

\section{Document Version}

Accepted author manuscript

Link to publication record in Manchester Research Explorer

\section{Citation for published version (APA):}

Romanis, E. C., \& Parsons, J. A. (2020). Legal and policy responses to the delivery of abortion care during COVID-19. International Journal of Gynecology and Obstetrics. https://doi.org/10.1002/ijgo.13377

\section{Published in:}

International Journal of Gynecology and Obstetrics

\section{Citing this paper}

Please note that where the full-text provided on Manchester Research Explorer is the Author Accepted Manuscript or Proof version this may differ from the final Published version. If citing, it is advised that you check and use the publisher's definitive version.

\section{General rights}

Copyright and moral rights for the publications made accessible in the Research Explorer are retained by the authors and/or other copyright owners and it is a condition of accessing publications that users recognise and abide by the legal requirements associated with these rights.

\section{Takedown policy}

If you believe that this document breaches copyright please refer to the University of Manchester's Takedown Procedures [http://man.ac.uk/04Y6Bo] or contact uml.scholarlycommunications@manchester.ac.uk providing relevant details, so we can investigate your claim.

\section{OPEN ACCESS}


ETHICAL AND LEGAL ISSUES IN REPRODUCTIVE HEALTH

Legal and policy responses to the delivery of abortion care during COVID-19

Elizabeth Chloe Romanis ${ }^{1, *} \&$ Jordan A. Parsons ${ }^{2}$

${ }^{1}$ Centre for Ethics and Law in the Life Sciences, Durham Law School, Durham University, UK

${ }^{2}$ Centre for Ethics in Medicine, Bristol Medical School, University of Bristol, UK

\section{${ }^{*}$ CORRESPONDENCE}

Elizabeth Chloe Romanis, Durham Law School, Durham University, UK.

Email: elizabeth.c.romanis@durham.ac.uk

\section{SYNOPSIS}

COVID-19 has exacerbated pre-existing barriers to abortion care globally. This paper highlights how legal and policy responses have protected or hindered equitable access to abortion.

\section{ABSTRACT}

Access to abortion care has long been a global challenge, even in jurisdictions where abortion is legal. The COVID-19 pandemic has exacerbated barriers to access, thereby preventing many women from terminating unwanted pregnancies for an extended period. In this paper, we outline existing and COVID-specific barriers to abortion care and consider potential solutions, including the use of telemedicine, to overcome barriers to access during the pandemic and beyond. We explore the responses of governments throughout the world to the challenge of abortion access during the pandemic, which are This article has been accepted for publication and undergone full peer review but has not been through the copyediting, typesetting, pagination and proofreading process, which may lead to differences between this version and the Version of Record. Please cite this article as doi: 10.1002/IJGO.13377

This article is protected by copyright. All rights reserved 
an eclectic mix of progressive, neutral, and regressive policies. Finally, we call on all governments to recognize abortion as essential healthcare and act to ensure that the law does not continue to interfere with providers' ability to adapt to circumstances and to guarantee safe and appropriate care not only during the pandemic, but permanently.

\section{KEYWORDS}

Abortion; Autonomy; COVID-19; Reproductive health; Reproductive rights; Sexual health; Telemedicine

\section{1 | INTRODUCTION}

The COVID-19 pandemic has made abortion access more difficult globally. Government policies of lockdown and social distancing, the burden on health services and staff redeployments, and damage to supply chains are making it increasingly difficult for women, including transgendered males and non-binary people that have the physiology to become pregnant [1], to access abortion services. Even where women can access inperson services, to do so they must put themselves and, by extension, those they live with, at risk of COVID-19 infection. In many cases these obstacles were pre-existing and have simply been exacerbated, whereas others have only recently arisen.

Abortion care is necessary to aid women seeking respite from unwanted and/or unsafe pregnancy [2], which has a significant impact on physical and mental health [3]. It is also necessary to mitigate the impact of unsafe abortion [4]. Consequently, abortion care is recognized as a human rights imperative - to protect the lives, bodily autonomy, and reproductive autonomy of women [2,3]. Sexual and reproductive health care remains crucial during the pandemic. Some have noted an increased likelihood of unwanted pregnancy due to present circumstances [3,5], thus amplifying the need to ensure access to care. The World Health Organization (WHO) notes that 'reductions in the availability of essential SRH [Sexual and Reproductive Health] and MNH [Maternal and Newborn Health] Services will result in many thousands of maternal and newborn deaths due to 
millions of additional unintended pregnancies, unsafe abortions and complicated deliveries without access to essential and emergency care' [6]. Despite abortion care being necessary to ensure the health and safety of many women, the pandemic has exacerbated existing disparities in access that exist in many countries globally. In some instances, this is the direct result of existing or newly implemented legal and policy barriers to care. In this paper we consider some of the ways in which abortion care might be appropriately facilitated in pandemic circumstances by providers adapting, for example by providing early medical abortion (EMA) through telemedical services (TEMA). The terms 'telemedicine' and 'telehealth' (and, to a lesser extent, 'e-health') are often used interchangeably. Strictly speaking, telemedicine is a sub-category of telehealth that specifically concerns clinical care. As our focus in this paper is on clinical care (i.e. medical abortion provided by healthcare professionals), we use the term telemedicine. We note that some transnational abortion providers that enable women to receive 'pills by post' may not consider themselves to be offering 'telemedical' services. This paper focuses on both the legal and policy context that existed pre-COVID-19 and the barriers and innovations that have been implemented in response to the pandemic that have either limited or facilitated the provision of (remote) abortion care.

In countries where TEMA is not locally available, it is important to note that women may still be able to access remote services due to the work of non-profit international organizations. Examples include Women on Web [7] and Women Help Women [8], which both continue to use the internet to provide abortion medication to women in countries where local access is challenging, either because it is legally restricted or difficult to access. We will refer to these organizations as transnational abortion providers.

\section{2 | BACKGROUND}

EMA is the use of two drugs-mifepristone and misoprostol-taken 24-48 hours apart to procure an abortion. This drug regimen, however, is not universally approved.

Mifepristone was first approved for use in some countries (including China, Spain, and the UK) in 1988, has only been approved in some countries in 2020 (including Cameroon 
and Chile), and remains prohibited elsewhere (including Brazil, Myanmar, and Slovakia) [9].

The effect of the drugs is an induced miscarriage and the expulsion of the products of conception. The safety, effectiveness, and acceptability of the procedure are well established. As such, it is recommended by the WHO for use up to nine weeks of gestation [10]. Different jurisdictions have slightly different gestational limits, though they are generally around the 10-week mark. EMA has been praised for its ability to enable self-managed abortion, and to drastically decrease maternal morbidity and mortalityparticularly in the global south [11].

TEMA is the use of telemedical means to provide EMA remotely. TEMA has been in practice in some countries for over a decade. However, there is substantial variation in the models adopted, and what is generally thought of as TEMA today-a consultation by telephone or video call before abortifacients are posted to the patient-is a more recent development. Whilst there are small differences between each TEMA service, there are some common models that have developed.

Some services have required-and continue to require-that women attend some sort of collaborating healthcare facility to obtain tests and ultrasounds. Results are then reviewed by an offsite physician and a telephone or video consultation takes place. If prescribed, drugs are then either dispensed by the healthcare facility (as with Planned Parenthood of the Heartland in North-Central US) or posted to the patient (as with the TelAbortion Project in the US). Whilst these services undoubtedly improve access, they do not overcome the barriers we will outline below for all women due to the in-person requirements.

By removing all in-person requirements, the services offered globally by transnational abortion providers further improve access. Following the completion of an online 
questionnaire-based consultation, abortifacients are posted to women as appropriate. Such services, however, are still limited in their reach for reasons we will explain later in this paper.

More recently —and in response to the pandemic - the British Pregnancy Advisory Service (BPAS - the leading provider of abortion services in England and Wales) introduced what can be considered a middle ground model between those already outlined. Women undergo a telephone consultation and, assuming doctor approval, are posted a pack including the necessary drugs and information [12]. This, we argue, is a preferable model as it combines the ease of an entirely remote service with the safeguards of a full, live consultation. However, we acknowledge that a variety of models will be necessary to meet the needs of individual countries. In areas where women may not have access to an internet connection or private telephone, a system involving the collaboration of local healthcare facilities which are not authorized to prescribe abortifacients themselves may be more appropriate.

\section{3 | ABORTION CARE DURING COVID-19}

\subsection{Practical barriers to in-person abortion care}

Those seeking to access abortion services frequently face practical barriers. Perhaps the most common, and one which is fed into by many others, is the distance to the nearest clinic. So-called "abortion deserts" in the US are frequently discussed [5], but a similar situation is mirrored across the world, particularly in countries with vast rural areas. Having to travel far to access care is not feasible for some, particularly if public transportation is limited and they have no access to a private vehicle. In that sense, socioeconomic status can challenge access to abortion. Geographical barriers are especially problematic where both medications must be taken under medical supervision, as per "traditional" EMA. As one of us has previously argued, there is no reason why home use of mifepristone and misoprostol should not be permitted, though some countries have been slow to allow this, let alone TEMA [13]. These medications can act 
very quickly, meaning that where the taking of medication is required to be supervised there is a risk of the induced miscarriage beginning on the journey home, such that living far from a clinic increases the likelihood of this.

Having to attend a clinic in person may also be challenging for those who work full-time, have children, and/or care for other relatives [5]. This is the case regardless of the distance to the nearest clinic. Taking time off work and arranging alternative care for dependents may not be immediately feasible. For women who, for whatever reason, do not want others to know that they are having an abortion, this may be even more difficult. Whereas a manager, for example, may be sympathetic and allow an employee time off work to attend an abortion clinic, this would require that employee to share this personal information. Where a pregnant woman cannot make such arrangements to seek an abortion soon after having made the decision, she may be forced to access treatment later in her pregnancy. Depending on timings, in some cases this may result in exceeding the legally permitted gestational limit in that woman's jurisdiction.

\section{2 | Pandemic exacerbation}

The above issues are longstanding. However, the COVID-19 pandemic has exacerbated them, thereby amplifying the case for TEMA. Whereas responses to the pandemic vary between countries, the vast majority have introduced some form of social distancing or lockdown. These measures make it more challenging for those seeking abortion services to get to a clinic even if they live within close proximity. With schools closed, caring responsibilities have increased for parents. Having to manage childcare along with, in some cases, the move to at-home working, can make it difficult to leave the house alone. Even for those who can, and those who do not have caring responsibilities there has been, and in many cases, there remains, an expectation to stay at home. Leaving the home presents a risk of infection that many pregnant women may be unwilling to take, particularly if they or someone they live with has an underlying health condition. In some cases, there may also be disruption to public transportation services. 
Similar issues face those who work at abortion clinics. Staff may struggle to get to work or may even fall ill themselves at a greater rate than prevails in their community. Marie Stopes International have noted that 'both support and frontline staff have been unable to work, due to movement restrictions, illness [and] family demands' [14]. The impact of this in some places could (and has) force(d) some clinics to close temporarily. Where this happens, the distance to the nearest clinic would increase for some patients. In Great Britain, BPAS announced in March 2020 that $23 \%$ of its clinics had closed because of the pandemic [15]. There are concerns about the health and safety of both staff and service users where clinics stay open.

All these obstacles risk preventing, or at the very least delaying, access to essential abortion services. This is problematic due to the time-sensitive nature of abortion, both medically and politically. We know that even though EMA is feasible past 12 weeks of gestation [10], the risk of incomplete abortion and various other complications increases with time. If a pregnancy is going to be terminated, the earlier this is done the safer it is. Furthermore, in some places it is far more difficult to find clinics that provide later-term abortions even where this is legal [16]. The legal obstacle also increases later in pregnancy, with late termination generally requiring an immediate health threat. Whilst these legal barriers, we suggest, are inappropriate, physicians are bound by them. There is, then, a risk that a continued requirement for EMAs to be provided in person throughout the pandemic will cause some pregnancies to exceed time limits, resulting in either the necessity for more invasive, surgical means of abortion, or an outright denial of care. For example, it is estimated that during the disruption of lockdown in India 1.85 million abortions-equating to $47 \%$ of the total anticipated during the period-were compromised as a result of, among other reasons, limits on the mobility of those seeking care [17].

Failure to ensure service provision has a 'disparate impact on those with low or no incomes and or who lack housing, migrants, refugees, people with disabilities and 
adolescents and compelling pregnancy worsens health outcomes, particularly in the case of COVID-19' [3]. We have also argued elsewhere that the impact of barriers resulting from COVID-19-controlling measures are more likely to be experienced by people who are already structurally disadvantaged, have a disability which means that they feel less safe leaving the home, or have caring responsibilities [5]. There are concerns that vulnerable women who are victims of domestic violence and coercive control, or who are living in communities and cultures in which abortion is heavily stigmatized, will find abortion much harder to access as they struggle to leave the home without explaining to family members where they are going [5]. As a result of the impact of COVID-19 on service provision, Todd-Gher and Shah have argued that 'incorporating measures to ensure safe abortion services into state pandemic responses and eliminating barriers to abortion is not just a matter of harm reduction - it is a human rights imperative' [3].

\section{3 | TEMA and other solutions}

TEMA is one important way to facilitate continued access to abortion care in pandemic conditions and expand access to groups that have previously had limited access. TEMA can overcome some of the most substantial barriers to care by enabling women to access care at home, thereby preventing them from having to travel (potentially long) distances and increasing their exposure to COVID-19. It also enables women to access care conveniently and comfortably and, in the pandemic context, protects healthcare providers from unnecessary exposure. TEMA has been found consistently to be safe, effective, and acceptable to women [18]. Because TEMA is safe, and because it can ensure access during this time, we have argued elsewhere that provision of this service is necessary [5]. In some places TEMA is being safely facilitated by local/national providers including Colombia, Great Britain, South Africa, and parts of the US.

Whilst TEMA can address some of the barriers to care at this time from the perspective of the woman accessing care, there may be some difficulty experienced by providers where legal restrictions prevent task-sharing in abortion care. Even though there is increasing evidence that nurses and other healthcare providers can safely prescribe abortifacients 
[19], in many jurisdictions the law dictates that only doctors can prescribe the medications, for example in Great Britain [20]. This raises some problems for providers in that it limits the provider pool and increases both waiting times and the cost of providing care [19]. The WHO recommends that task-sharing be adopted in the delivery of health services to optimize delivery [21,22]. During the pandemic (and beyond), permitting, for example, nurse prescribing would simplify access and reduce administrative burdens on doctors, thereby enabling doctors to focus on cases that involve more complex management. There is some extent to which regional providers have already attempted to adopt task-sharing as far as is possible-for example, BPAS provides initial consultations with nurse-midwives, including the obtaining of patient consent, before doctors review the case and sign off on the prescription [23]. However, this model still has many unnecessary administrative hurdles, including requiring doctors' signatures in straightforward cases.

In some instances, it may be that there are significant logistical barriers to the implementation of TEMA. For example, some women who need access to abortion care have limited access to the internet, such that other solutions in ensuring access to the safest possible in-person care at this time may be more appropriate. To maximize the safety of in-person abortion care, protocols have been developed for 'no test/no touch' treatment [24]. Without performing physical examinations and/or ultrasounds, providers can still reduce the risk of transmission between themselves and patients.

\section{4 | LEGAL AND POLICY CONTEXT OF ABORTION CARE DURING COVID-19}

Some of the most significant barriers to abortion care during COVID-19 globally exist at the local level; in some countries there are restrictions which mean that while abortion is legal, the provision of remote abortion (by telemedicine) is not. These barriers take various forms and, in many cases, restrictions on service provision were in place before the pandemic. This meant that to address access issues that have arisen during COVID19 , a change to the law to remove particular restrictions became necessary. These restrictions limit the capacity of care providers to respond to situations at hand to ensure 
that they meet their professional responsibility to ensure patient wellbeing and access to care.

First, in some countries there are explicit bans on the provision of TEMA. For example, some states in the United States (including Arizona, Arkansas, Indiana, South Carolina, and West Virginia) [5]. Some countries have also taken action to limit the assistance that might be provided to women by transnational abortion providers; the criminal process is sometimes utilized to punish those who are caught accessing care this way (this was a particular drive for aggressive prosecutions in Northern Ireland prior to the decriminalisation of abortion in 2019) [25]. In Spain, action has been taken to block the websites of these providers [26, 27].

Second, in some countries there are other restrictions which, whilst not an explicit ban on TEMA or remote care itself, have the effect of rendering it inaccessible because other legal requirements necessarily preclude the possibility of care being facilitated entirely remotely. For example, the legal requirement that a pregnant person has an ultrasound, or some form of physical examination, before taking abortifacients. This is the case in Austria, Tunisia, and some states in the United States (including Alabama, Louisiana, Mississippi, Missouri, Nebraska, Oklahoma, South Dakota, and Texas) [5]. Other procedural requirements embedded in abortion law in some countries, such as mandatory counselling or 'reflection delays' [28], might not necessarily preclude remote care, but still constitute a significant barrier to timely and safe access. In Spain (except for Catalonia) it is required that women collect information about abortion in person to reflect on during a mandatory waiting period before receiving treatment [27].

Some countries have placed specific regulations on EMA medications that render remote abortion care unavailable without constituting explicit bans on TEMA. In Great Britain, until recent legal changes [5], women had to be supervised taking the first abortion medication, mifepristone, in a clinic [19]. Legal requirements, in place before the 
pandemic, that one or both EMA medications must be administered in a clinic, are widespread globally. In Italy, the law specifies that abortions must take place under the service of obstetrics and gynaecology in a general hospital, or at specialist public hospitals and private clinics [29]. In Iceland, the law specifies that abortion must be provided in a hospital or healthcare facility, but medical abortion could also be provided in doctors' offices regulated by the Medical Director of Health and Public Health [30]. In Ghana, the Criminal Code similarly states that abortion is permissible only when provided by a registered medical practitioner in a government hospital, a registered private hospital or clinic, or a place approved for the purpose by legislative instrument [31]. In Cambodia, abortion may be carried out in a hospital, health centre, or clinic which is authorized by the Ministry for Public Health, which must have the capacity to provide emergency medical treatment and means of transport to hospital [32]. Similar laws are in place in several US states (including North Carolina, North Dakota, Tennessee, and Wisconsin) [5]. There are also policies by pharmaceutical regulators that further complicate the remote provision of these medicines. In the US, for example, the Food and Drug Administration (FDA) stipulates that mifepristone should be dispensed only in clinics, medical offices, and hospitals, or under the supervision of an 'FDA-certified' prescriber [33].

Finally, in some countries legal and policy barriers to (remote) abortion care have been erected by governments and local administrations directly in response to the pandemic. Such policy changes are discussed in the next section.

\subsection{Regulation and policy changes during COVID-19}

We have seen varied responses across jurisdictions to the challenges to abortion access during the pandemic. Those seeking abortions-like everyone else-have been asked to minimize in-person contact with others, which some countries have supported by implementing remote access to abortion care. Others, however, have seized the opportunity for regressive policy by either actively or effectively banning abortion in the circumstances. 
Todd-Gher and Shah argue that states should 'introduce bold, innovative measures to maintain and expand access [to abortion care] in accordance with human rights' [3]. The Center for Reproductive Rights has also called for the implementation of telemedicine in reproductive health services to ensure that 'women and girls are not unnecessarily compelled to make multiple trips to healthcare facilities' [34]. Further, in its COVID-19 guidance on maintaining essential health services, the WHO has recommended enabling safe abortion to the full extent of the law and to 'minimize facility visits and provider-client contacts through the use of telemedicine [emphasis added] and self-management approaches' [6]. Some countries have acted (temporarily) to remove existing legal barriers to TEMA, namely a requirement that women attend a clinic in person to receive and/or take abortion medication, in response to the pandemic. In Great Britain, for example, abortion regulation was specifically relaxed in England, Wales, and Scotland to allow abortion providers to begin a 'pills by post' service [5]. In Ireland, there was a mandatory requirement that women attend two appointments with their primary care provider three days apart to access abortion, and the law specified that abortion may be carried out where the medical practitioner is satisfied that the pregnancy is less than 12 weeks of gestation 'having examined the pregnant woman' [35]. The Health Minister in Ireland, Simon Harris, announced that they did not believe this provision excluded the possibility of examination by remote means [36], and the Health \& Safety Executive introduced a model of remote service provision in March 2020, though noting that this was 'only... for the duration of the pandemic...' [37].

In France, the health administration has, in response, both enabled service providers to offer remote consultation and temporarily increased the gestational limit for selfadministration at home from seven to nine weeks [38]. In South Africa, telemedicine had been previously used in abortion follow-up care [39], but the law on telemedicine technically requires that there be a pre-existing relationship between patient and professional. However, following successful campaigning by the Sexual and Reproductive Justice Coalition, Marie Stopes International have been enabled to offer TEMA during the pandemic [40]. Telemedicine has also now been permitted for pre- 
abortion counselling in Germany (provided by an independent third party as per the requirements of the German Criminal Code), which is a step in the right direction, despite the fact that abortion care must still be administered in a clinic [27]. There have also been some successful legal challenges to policies that prevent access at this time. For example, in Maryland in the US, the FDA requirement that mifepristone be dispensed in person was recently successfully challenged, and an injunction is in place (at the time of writing) that prevents the FDA from enforcing this requirement in Maryland [41].

Other countries, however, have failed to act appropriately to (temporarily) remove preexisting legal barriers to TEMA implicitly labelling abortion services as non-essential in failing to adapt. In some countries, governments have attempted to exploit the pandemic to effectively ensure access to abortion at clinics is even more difficult by explicitly adopting measures that label abortion care 'non-essential'. In the US, 11 states have issued statements at some point during the pandemic that abortion was 'non-essential care' and thus must be suspended to 'divert resources to the front line' [42]. Rebouché argues that it is more likely that restricting abortion access will result in greater strain on the health system because the alternative to TEMA, or 'no-touch [early medical] termination' because more people will need assistance after inducing their own abortions, and advanced pregnancy, childbirth and neonatal care will require more resources [43]. This was an attempt to force abortion clinics to close and was also the approach taken by authorities in European countries including Austria, Croatia, Germany, and Romania, which did not recognize abortions as essential [42]. Such an approach has the effect of banning abortion in reality, even if not in name [5]. In some countries, governments have gone further than labelling abortion non-essential. In Slovakia (where only surgical abortion is lawful), the government has released statements that it 'does not recommend' women accessing care at this time [44]. In Poland, the government has taken steps to pass even more regressive regulation, even further limiting access, at a time when it is more difficult for effective citizen protest to be organized [45]. 
Regulation and policy change that effectively reduces the availability of abortion in the short term due to COVID-19 is a major concern. This puts many women at risk, forcing them to endure the substantial harms associated with unwanted pregnancy [2,3] and/or face a riskier procedure in procuring an abortion through legal channels when services resume. The non-essential classification of abortion in some places has neglected the time-sensitivity of abortion; women cannot wait long before the option of EMA is removed and surgical abortion becomes necessary, or they reach the point at which abortion of any form is impermissible by law (depending on the legal framework of the country concerned). Further, such classification fails to recognize that the risk of complications increases with gestational age and, therefore, delaying access is both physically and mentally harmful. In some places, women may feel forced to resort to riskier methods of abortion to avoid an unwanted pregnancy, potentially putting them at risk of criminal sanction and/or endangering their health and life.

\section{2 | The lasting impact of legal and policy changes in response to COVID-19}

The over-regulation (both in law and by professional regulators) of abortion medication has the impact of limiting the ability of providers to facilitate remote provision. This clearly will have had and continues to have a significant effect on individual women because they might find accessing care more difficult at this time. Furthermore, this overregulation has some wider social impacts and perpetuates broader harms to women. Law is a medium through which stigma about women, their capacities, and their choices is constructed and enforced [46]. The requirement in some places that people accessing EMA are 'supervised' swallowing mifepristone (and misoprostol) in clinics, when this is not grounded in medical necessity [18], perpetuates the view that women are irresponsible, not trustworthy, and/or are incapable of making their own decisions about abortion. Being mindful of the harm that law can generate in its operation [47], laws that mandate supervision infantilize and construct women as subjects who need protection from themselves and their own care. In the context of the pandemic, singling out this care as something that cannot occur in the home, as other areas of healthcare are 
increasingly provided in this way, further perpetuates abortion stigma. Such approaches also further bolster the narrative that is often adopted and perpetuated by anti-choice groups that abortions are generally unsafe when we know this is not the case. It also increases the likelihood that women will access care outside of the health system, which is riskier.

The progressive changes in countries like France, Great Britain, and Ireland must lead to a permanent relaxation of abortion restrictions if pregnant women's health and rights are to be respected, protected, and fulfilled $[3,5]$. However, this would still require further positive action in some cases. The changes in England and Wales are temporary-and specifically have a sunset clause - so further campaigning is likely to be necessary. Though if the evidence base that emerges from this period demonstrates, as TEMA services elsewhere have demonstrated, that service provision in this form is safe, effective, and acceptable to service users [18, 48], it will be more difficult for governments to avoid discussing permanence. The changes in some places being explicitly labelled as temporary (and in England and Wales formalized as such with legal mechanisms) is, however, actively unhelpful in this regard as it perpetuates the need for caution around the process and effectively prevents the embracing of organized services that allow selfmanagement. Sheldon has observed that UK providers 'often find themselves caught in the tension between offering reproductive choice and the best possible care for the women they treat yet being constrained to abide by the highly medicalized model of control entrenched in statute' [49], and which no longer reflects best practice. Progressive policy changes during COVID-19, however, might further the case for change by demonstrating their utility.

The lasting impact of COVID-19 on abortion care in places that have not adopted progressive responses should be cause for concern. For example, in parts of the US and Poland, the state of emergency currently faced has been used to further an outdated political agenda that neglects the health of women by labelling abortion 'non-essential'. In these countries, a disruption in access, or providers being forced to direct their attention 
to the fight to stay open, has limited women's access substantially. Moreover, labelling abortion as non-essential runs the risk of reversing the work of providers and reproductive rights groups in establishing the essential nature of abortion provision globally. This could embolden the rhetoric of groups or politicians seeking to limit access to care. In Poland, as explained, this culminated in an attempt to introduce even more restrictive legislative provisions restricting abortion care in April 2020 [45]. Such restrictions, in Poland and beyond, would threaten the lives and health of millions of women.

\section{5 | CONCLUSION}

That government responses to the challenges to abortion access created by the COVID19 pandemic are so varied demonstrates that there is still a way to go until abortion is universally recognized as essential healthcare. Even where TEMA has been enabled in response to COVID-19, it is usually a temporary provision that is intended to be revoked as we emerge from the pandemic. Whilst it is still positive that such provisions have been made, it is important, moving forward, for temporary policies enabling remote access to abortion care, and other such measures that facilitate access and allow service providers to evolve and implement best practice [19], to be made permanent. Furthermore, even in these jurisdictions there remain some legal and policy barriers that prevent care providers from adapting to the circumstances-for example, in Great Britain two doctors' signatures remain necessary for abortion [19], and the FDA continues to place more stringent requirements on prescription of mifepristone compared to other medications in the US [33]. As COVID-19 continues to impact on care now and into the future it is important that providers continue to play an important role in ensuring access to care and improving this in line with best practice. This will include, as many service providers are doing, documenting the safety and efficacy of TEMA [50] and reflecting on what has (and potentially has not) worked in service development, and providing expert advice to policymakers and ethics committees about how legal barriers and policies interfere with the implementation of best clinical practice. The International Federation of Gynecology and Obstetrics (FIGO) recommends that member societies should advocate against restrictions on sexual and reproductive rights in government policies in response to 
COVID-19 [51]. This includes being mindful of the disproportionate impact that barriers to abortion care have on those who are already marginalized in accessing health care as a result of their socio-economic status or location.

In some jurisdictions, it is clear that governments have monopolized on the circumstances to limit access to abortion, either by failing to remove existing legal barriers that in the circumstances prevent providers from adapting or by actively labelling abortion services as 'non-essential'. We have noted with concern how this might do lasting damage to sexual and reproductive rights in these jurisdictions. It is essential that access to abortion resume in those jurisdictions that are either still actively or effectively preventing abortion care at this time.

\section{REFERENCES}

1. Dickens BM. Transsexuality: Legal and ethical challenges. Int J Gynecol Obstet 2020. doi:10.1002/ijgo.13307

2. Hervey T, Sheldon S. Abortion by Telemedicine in the European Union. Int $J$ Gynecol Obstet. 2019;145:125-128.

3. Todd-Gher J, Shah PK. Abortion in the context of COVID-19: a human rights imperative. Sex Reprod Health Matters. 2020; [Epub ahead of print].

4. Singh S, Maddow-Zimet I. Facility-based treatment for medical complications resulting from unsafe pregnancy termination in the developing world, 2012: a review of evidence from 26 countries. BJOG. 2016;123:1489-1498.

5. Romanis EC, Parsons JA, Hodson N. COVID-19 and Reproductive Justice in Great Britain and the United States: Ensuring Access to Abortion Care during a Global Pandemic. J Law Biosci. 2020; [Epub ahead of print].

6. World Health Organization. Maintaining essential health services: operational guidance for the COVID-19 context [WHO website]. 2020. 
https://www.who.int/publications/i/item/10665-

332240?utm_source=International\%20Campaign\%20for\%20Women $\% 27$ s\%20Right $\%$ 20to\%20Safe\%20Abortion\%20membership\%20list\&utm_campaign=062f9afcdb\&utm_medium=email\&utm_term=0_c9f67cdfa6-062f9afcdb-64323401. Accessed June 25, 2020.

7. Women on Web. https://www.womenonweb.org/en/. Accessed 24 August 2020.

8. Women Help Women. https://womenhelp.org/. Accessed 24 August, 2020.

9. Gynuity Health Projects. Map of Mifepristone Approvals [Gyniuity Website]. 2020. https://gynuity.org/assets/resources/mapmife-en.pdf. Accessed 20 August, 2020.

10. World Health Organization. Safe abortion: technical and policy guidance for health systems, Second edition [WHO website]. 2012.

http://apps.who.int/iris/bitstream/handle/10665/70914/9789241548434_eng.pdf;jsessi onid=1524B2E3691EF62076BDC8103BEAE707?sequence=1. Accessed June 25, 2020.

11. Jelinska K, Yanow S. Putting abortion pills into women's hands: realizing the full potential of medical abortion. Contraception. 2018;97:86-89.

12. British Pregnancy Advisory Service. Pills by Post - Abortion Pill treatment at home [BPAS Website] 2020. https://www.bpas.org/abortion-care/abortion-treatments/theabortion-pill/remote-treatment/. Accessed September 1, 2020.

13. Parsons JA. 2017-19 governmental decisions to allow home use of misoprostol for early medical abortion in the UK. Health Policy. 2020;124:679-683.

14. Church K, Gassner J, Elliott M. Reproductive health under COVID-19 challenges of responding in a global crisis. Sex Reprod Health Matters. 2020; [Epub ahead of print].

15. British Pregnancy Advisory Service. Healthcare professionals call on Boris Johnson to intervene to protect women's health - reckless failure to listen to scientific advice is putting vulnerable women at severe risk [BPAS Website]. March 25, 2020. https://www.bpas.org/about-our-charity/press-office/press-releases/healthcare- 
professionals-call-on-boris-johnson-to-intervene-to-protect-women-s-health-recklessfailure-to-listen-to-scientific-advice-is-putting-vulnerable-women-at-severe-risk/. Accessed on June 25, 2020.

16. Ruggiero S, Brandi K, Mark A, et al. Access to later abortion in the United States during COVID-19: challenges and recommendations from providers, advocates, and researchers. Sex Reprod Health Matters. 2020. [Epub ahead of print].

17. Ipas Development Foundation. Compromised Abortion Access due to COVID-19: A model to determine impact of COVID-19 on women's access to abortion [Ipas Website]. 2020.

https://www.ipasdevelopmentfoundation.org/resourceFiles/59202006092650.pdf. Accessed June 25, 2020.

18. Endler M, Lavelanet A, Cleeve A, Ganatra B, Gomperts R, Gemzell-Danielsson K. Telemedicine for medical abortion: a systematic review. BJOG. 2019;126:1094-1102.

19. Lohr P, Lord J, Rowlands S. How would decriminalisation affect women's health? In: Sheldon S, Wellings K, eds. Decriminalising Abortion in the UK: What Would it Mean? Bristol: Policy Press; 2020:37-56.

20. Abortion Act 1967, s.1 (Great Britain).

21. World Health Organization. WHO Recommendations: Optimizing Health Worker Roles to Improve Access to Key Maternal and Newborn Health Interventions Through Task Shifting [WHO website]. 2012. https:// optimizemnh.org/optimizing- healthworker- roles- maternal- newborn- health/. Accessed August 10, 2020.

22. Sorhaindo AM, Ganatra B. Expanding health worker roles and decentralizing safe abortion and postabortion care: Experiences in diverse settings. Int J Gynecol Obstet 2020;150:1-3.

23. British Pregnancy Advisory Service. 2020. Coronavirus (COVID-19 essential information) [BPAS Website]. 2020. https://www.bpas.org/contact-us/covid-19. Accessed 24 August 2020. 
24. Raymond E, Grossman D, Mark A, et al. Commentary: No-test medication abortion: A sample protocol for increasing access during a pandemic and beyond. Contraception. 2020; 101:361-366.

25. Fox M, Horgan G. The effects of decriminalisation in Northern Ireland. In: Sheldon S, Wellings K, eds. Decriminalising Abortion in the UK: What Would it Mean? Bristol: Policy Press; 2020:77-98.

26. Women on Web. June 17, 2020. Spain censors information about abortion amid Covid-19 lockdown. https://www.womenonweb.org/en/page/20230/spain-censorsinformation-about-abortion-amid-covid-19-lockdown. Accessed 24 August, 2020.

27. European Parliamentary Forum for Sexual \& Reproductive Rights and IPPF European Network [IPPF Website]. April 22, 2020. Sexual and Reproductive Rights during the COVID-19 pandemic. https://www.ippfen.org/sites/ippfen/files/202004/Sexual\%20and\%20Reproductive\%20Health\%20during\%20the\%20COVID19\%20pandemic.pdf. Accessed 20 August, 2020.

28. Rowlands S, Thomas K. Mandatory Waiting Periods Before Abortion and Sterilization: Theory and Practice. Int. J. Women's Health. 2020;12:577-586.

29. Law 194 of the Italian Republic, 1978, article 8/9.

30. Law on abortion 2019, 7 gr (Iceland).

31. Acts of Ghana Criminal Code, 1960 (Act 29), s.58.

32. Cambodia Law on Abortion 1997, article 6.

33. Mifeprex REMS Study Group. Sixteen Years of Overregulation: Time to Unburden Mifeprex. N Engl J Med 2017; 376:790-794.

34. Center for Reproductive Rights. June 4, 2020. In the Face of the COVID-19 Pandemic, Sexual and Reproductive Health Services are Essential. https://reproductiverights.org/press-room/face-covid-19-pandemic-sexual-andreproductive-health-services-are-essential. Accessed June 29, 2020.

35. Health (Regulation of Termination of Pregnancy) Act, s.12 (Ireland). 
36. Valerie Ryan for the Irish Medical Times. March 27, 2020. Telemedicine abortion consultations permitted - Health Minister.

https://www.imt.ie/uncategorised/telemedicine-abortion-consultations-permittedhealth-minister-27-03-2020/. Accessed 10 August 2020.

37. Kitty Holland for The Irish Times. March 27, 2020. Coronavirus: Concern crisis could prevent timely access to abortion services.

https://www.irishtimes.com/news/social-affairs/coronavirus-concern-crisis-couldprevent-timely-access-to-abortion-services-1.4213703. Accessed 10 August, 2020.

38. High Authority for Health. April 10, 2020. Drug-induced Voluntary Interruption of Pregnancy (IVG) at the 8th and 9th week of amenorrhea (AS) outside a hospital setting. http://www.has-sante.fr/jcms/p_3178808/fr/interruption-volontaire-degrossesse-ivg-medicamenteuse-a-la-8eme-et-a-la-9eme-semaine-d-amenorrhee-sahors-milieu-hospitalier. Accessed 11 August, 2020.

39. Constant D, de Tolly K, Harries J, Myer L. Assessment of completion of early medical abortion using a text questionnaire on mobile phones compared to a selfadministered paper questionnaire among women attending four clinics, Cape Town, South Africa. Reprod Health Matters. 2014;22:83-93.

40. Marion Stevens for International Campaign for Women's Right to Safe Abortion. May 1, 2020. South Africa: a space of many contradictions - and now Covid-19. https://www.safeabortionwomensright.org/blog/south-africa-a-space-of-manycontradictions-and-now-covid-19/. Accessed June 29, 2020.

41. Jones RK, Lindberg L, Witwer E. COVID-19 Abortion Bans and Their Implications for Public Health. Perspect Sex Reprod Health. 2020; [Epub ahead of print].

42. Martina Stevis-Gridneff, Alisha Haridasani and Monka Pronczuk for the New York Times. June 14, 2020. Coronavirus Created an Obstacle Course for Safe Abortions. https://www.nytimes.com/2020/06/14/world/europe/coronavirus-abortionobstacles.html. Accessed 10 August, 2020. 
43. Rebouché R. Assuring Access to Abortion. In: Burris S, de Guia S, Gable L, Levin DE, Parmet WE, Terry NP, eds. Assessing Legal Responses to COVID-19. Boston: Public Health Law Watch; 2020:117-122.

44. Zuzana Gabrižová for EURACTIV. April 29, 2020. COVID-19 restricts access to abortions in Slovakia. https://www.euractiv.com/section/politics/news/covid-19restricts-access-to-abortions-in-slovakia/. Accessed 15 August, 2020.

45. Shaun Walker for The Guardian. Concerns over Polish government tightening abortion laws during Covid-19 crisis. April 15, 2020.

https://www.theguardian.com/world/2020/apr/14/concerns-over-polish-governmenttightening-abortion-laws-during-covid-19-crisis. Accessed June 29, 2020.

46. Burris S. Stigma and the Law. Lancet. 2006; 367: 529-531.

47. Smart C. Feminism and the Power of Law. London: Routledge; 1989.

48. Aiken ARA, Digol I, Trussell J, Gomperts R. Self reported outcomes and adverse events after medical abortion through online telemedicine: Population based Study in the Republic of Ireland and Northern Ireland. BMJ. 2017; 357:j2011.

49. Sheldon S. The Medical Framework and Early Medical Abortion in the U.K.: How Can a State Control Swallowing? In: Cook R, Erdman J, Dickens B, eds. Abortion Law in Transnational perspective: Cases and Controversies. Philadelphia: University of Pennsylvania Press; 2014:189-209.

50. Bateson D, Lohr P, Norman WV et al. The impact of COVID-19 on contraception and abortion care policy and practice: experiences from selected countries. BMJ Sex Reprod Health. 2020. [Epub ahead of print].

51. FIGO Committee on Ethical and Professional Aspects of Human Reproduction and Women's Health [FIGO website]. July 2020. FIGO Ethics and Professionalism Guideline 080: Ethical Challenges of the COVID-19 pandemic.

https://www.figo.org/figo-ethics-and-professionalism-guideline-080-ethical-challengescovid-19-pandemic. Accessed on September 1, 2020. 